\title{
TRANSPORTE COLABORATIVO MARÍTIMO: UMA ANÁLISE SOB A ÓTICA DO MÉTODO SYSTEM DYNAMICS APLICADA À INDÚSTRIA MANUFATUREIRA
}

\section{COLLABORATIVE MARITIME TRANSPORTATION: AN ANALYSIS UNDER SYSTEM DYNAMICS METHOD APPLIED TO THE MANUFACTURING INDUSTRY}

\author{
Vanina Macowski Durski Silva ${ }^{1}$; Antonio Sérgio Coelho ${ }^{2}$; Antonio Galvão Novaes $^{3}$ \\ ${ }^{1}$ Universidade Tecnológica Federal do Paraná - UTFPR - Ponta Grossa - Brasil \\ vaninasilva@utfpr.edu.br \\ ${ }^{2}$ Universidade Federal de Santa Catarina - UFSC - Florianópolis - Brasil \\ coelho@deps.ufsc.br \\ ${ }^{3}$ Universidade Federal de Santa Catarina - UFSC - Florianópolis - Brasil \\ novaes@deps.ufsc.br
}

\begin{abstract}
Resumo
Este artigo tem como objetivo apresentar um modelo que analise os efeitos sistêmicos da política de colaboração entre indústrias manufatureiras que utilizam o transporte marítimo para a exportação. Pesquisou-se sobre a era da Logística Colaborativa e a Gestão do Transporte Colaborativo e, foram realizadas entrevistas com empresários e especialistas de modo a validar a proposta de estudo, além de obter dados para inserção no modelo. Posteriormente, foi realizado um estudo sobre os métodos Agent Based Modeling and Simulation e System Dynamics. O modelo proposto utilizando-se do SD contribuiu para a análise dos efeitos sistêmicos da política de colaboração entre indústrias manufatureiras, as quais se unindo fortalecem o poder de barganha sobre os armadores marítimos, influenciando na redução do preço de frete marítimo. Este trabalho contribuiu para o entendimento da importância de se adotar uma abordagem interdisciplinar para lidar com os problemas do transporte marítimo.
\end{abstract}

Palavras-chave: Logística; Transporte marítimo colaborativo; System Dynamics; Simulação.

\section{Introdução}

A gestão da cadeia de suprimentos atua na coordenação das múltiplas relações ocorrentes na cadeia, isto é, nas redes de organizações envolvidas em criar serviços e produtos ao consumidor final. De acordo com Novaes (2007), quando se fala em cadeia de suprimentos se pensa imediatamente no fluxo de materiais formado por insumos, componentes e produtos acabados. Esses fluxos entre os participantes da cadeia de suprimentos historicamente apresentam conflitos nos canais de negociação. Observa-se que cada elo busca minimizar seus custos individuais, o que 
normalmente não converge ao ótimo global da cadeia de suprimentos (SEIFERT, 2003). Dessa forma, no intuito de reduzir custos, aumentar eficiências e obter vantagens competitivas, verifica-se que as empresas estão sendo forçadas a repensar seus procedimentos, a utilizar técnicas de reengenharia e redefinir os relacionamentos e os modelos de suas cadeias de suprimentos. É neste contexto que emergiu o conceito global CPFR - Collaborative Planning, Forecasting and Replenishment (Planejamento, Previsão e Reabastecimento Colaborativo), no fim da década de 90. Esse conceito expressa a integração dos diversos participantes da cadeia de suprimentos, de modo a garantir aumento nas vendas, alinhamento inter-organizacional, eficiência operacional e administrativa. Posto isso, um dos campos de aplicação do CPFR se dá no transporte, recebendo a denominação de CTM - Collaborative Transportation Management (Gestão do Transporte Colaborativo). Observa-se que há um consenso entre os especialistas de que essa ferramenta apresenta um grande potencial para auxiliar nas reduções de custos e riscos, aumento no desempenho de serviço e capacidade, bem como na obtenção de uma cadeia de suprimentos mais dinâmica (SEIFERT, 2003; TACLA, 2003).

Neste contexto, percebeu-se uma lacuna ainda a ser explorada no que se refere à pesquisa para a resolução do problema de transporte colaborativo de cargas por parte das indústrias de manufatura utilizando-se do modal aquaviário. Assim sendo, este artigo tem como objetivo apresentar um modelo que analise os efeitos sistêmicos da política de colaboração entre indústrias manufatureiras que utilizam o transporte marítimo para a exportação, levando em consideração os principais parâmetros estratégicos e operacionais envolvidos nessa operação.

\subsection{Método de pesquisa e estrutura do trabalho}

A escolha do método foi iniciada com uma análise das diferentes possíveis abordagens para o problema proposto, através da revisão da literatura e determinação do objeto de estudo e, a partir daí concluiu-se que a abordagem mais adequada ao problema seria a do experimento através da modelagem. Após a modelagem do problema e realizadas simulações, o objeto de estudo foi validado levando à algumas conclusões e recomendações para estudos futuros.

Este artigo é composto de 6 seções, incluindo esta introdutória. No item 2 são apresentados alguns conceitos sobre a logística colaborativa e a gestão do transporte colaborativa. No item 3 é apresentado o mecanismo para a realização da exportação de produtos manufaturados através do transporte marítimo. No item 4 tem-se o modelo proposto para a análise do problema de transporte colaborativo e no item 5 apresentam-se os resultados da análise. Por fim, o item 6 apresenta algumas considerações finais e sugestões para continuidade do trabalho. 


\section{Logística colaborativa e o sistema de gestão do transporte colaborativo}

Desde a introdução do conceito de ECR - Efficient Consumer Response (Resposta Eficiente ao Consumidor) em meados de 1993, os participantes da cadeia têm tentado olhar além do seu próprio negócio de modo a tornar realidade o conceito de ECR, com todos os envolvidos trabalhando juntos através principalmente das redes de comunicações formadas pelo EDI (Silva et al, 2009). De acordo com pesquisa de Tacla (2003) não existe na literatura documento em abundância que ratifique o surgimento da fase da "logística colaborativa", mas é possível encontrar material denominando-a como a "nova onda". Iniciativas e trabalhos recentes apresentados em diversos congressos de renome, como a conferência do POMS - Production and Operations Management Society, do ano de 2008 já apresentam essa abordagem como um passo adiante na evolução do conceito de SCM. A iniciativa mais forte a conceituar a Logística Colaborativa chamase "CPFR" (Collaborative Planning, Forecasting and Replenishment) e pode ser vista como uma tentativa que almeja aumentar o fluxo de caixa e melhorar a performance dos retornos sobre os investimentos, além de melhorar a gestão do fluxo dos bens desde os produtores até os consumidores finais.

Similar ao CPFR, o Transporte Colaborativo envolve informações e fluxos de processos de fornecedores e compradores que colaboram juntamente com transportadores ou 3PL's para prover efetiva e eficiente entrega do carregamento. Conceitualmente as empresas podem ingressar no sistema de Transporte Colaborativo com ou sem o emprego do CPFR. Os processos de negócios mais geralmente associados com a colaboração da cadeia de suprimentos são aqueles envolvidos em CPFR. Entretanto, o Transporte Colaborativo tem sido referenciado como o "elo perdido" da execução da cadeia de suprimentos colaborativa. Sem a habilidade de desenvolver previsões efetivas de carregamento, as previsões de ordens que foram desenvolvidas pelo CPFR poderiam ser atendidas sem acuracidade; no entanto, o Transporte Colaborativo provê o próximo passo crítico após a geração de ordens via o CPFR (Sutherland, 2003). Ver Silva et al (2009) para maiores informações sobre como implementar o CTM.

Apesar da temática sobre transporte colaborativo ser relativamente nova, alguns trabalhos preliminares já a propuseram de maneira mais teórica (GOMBER et al, 1997; SCHÖNBERGER, 2005; BLOOS e KOPFER, 2009) e outros, de maneira mais aplicada, utilizando-se da programação matemática e simulação (CARNIERI et al, 1983; TACLA, 2003; GIESEN et al, 2007; NOVAES et al, 2009; SILVA et al, 2010-a, 2010-b, 2011-a, 2011-b, 2011-c). 


\section{Mecanismo para realização do transporte marítimo no caso da exportação de produtos manufaturados}

Conforme apresentado por Silva et al (2011-b), seguindo-se o fluxo em azul na Figura 1 a negociação se inicia através da indústria de manufatura (1) a qual pode atuar isoladamente sendo responsável por todos os arranjos através da cadeia de distribuição. Nesta situação a indústria manufatureira contrata o transportador terrestre (4) para transferir os produtos manufaturados da indústria ao porto. Há também a possibilidade ou, em muitos casos, a necessidade de primeiramente transferir os produtos manufaturados a um armazém (3) para manter um estoque que pode ser útil para resolver problemas de entregas rápidas ou para reter a carga até o momento em que todas as burocracias para exportar estão resolvidas. A indústria também é responsável por escolher o porto de origem a ser utilizado e ao mesmo tempo ela deve negociar com os armadores (7) o preço do frete marítimo.

Os fretes podem ser cotados como básicos, neste caso, comportando adicionais como taxas e sobretaxas. Neste estágio é comum contratar um NVOCC (6). Este agente é responsável por gerenciar a demanda de diversas indústrias por transporte marítimo de modo a negociar diretamente com os armadores o preço do frete e a disponibilidade de navios aos destinos dos produtos manufaturados das indústrias. No lado do destino há outra necessidade por parte das indústrias manufatureiras em relação à definição do porto de destino (8) a ser utilizado, que deve ser o mais apropriado de modo a entregar os produtos aos clientes (11).

Figura 1- Etapas do mecanismo de exportação

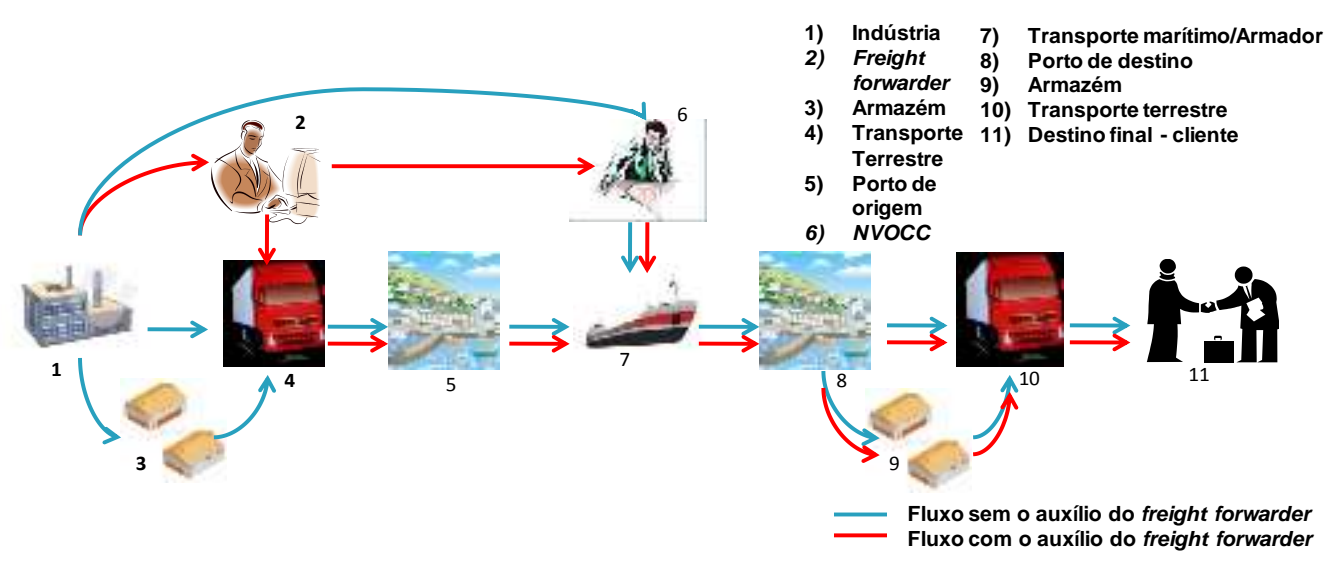

Fonte: Silva et al (2011-a)

Dando sequiência no fluxo de exportação, para efetuar as entregas as indústrias manufatureiras devem também contratar transportadores terrestres (10) para transportar seus produtos a armazéns intermediários (9) ou aos destinos finais no país destino. O fluxo em vermelho na Figura 1 é quase o mesmo que o fluxo azul exceto pelo fato que há a presença do freight 
forwarder (2). Neste caso a indústria manufatureira contrata este agente para ser o responsável por contratar e controlar todos os estágios da cadeia de distribuição. Esta é normalmente uma prática adotada por pequenas e médias indústrias que não possuem expertise em tal processo e portanto, o freight forwarder, quem gerencia a demanda de diversas indústrias por transporte marítimo, pode ser ágil na negociação.

\section{Modelagem do problema de transporte colaborativo}

Dentre as metodologias de experimento, a modelagem foi escolhida para este trabalho e a técnica computacional adotada foi a simulação. Posto isso, foi realizado um levantamento bibliográfico sobre o método de simulação Agent based Modeling and Simulation (ABMS), analisadas suas principais características e aplicações bem como iniciado o processo de modelagem do problema de estudo. Posteriormente, em discussão com especialistas e pesquisadores, foi levantada a hipótese de utilizar o método de simulação System Dynamics (SD) para a modelagem do problema de transporte colaborativo e novo levantamento bibliográfico foi realizado, bem como analisadas as possíveis aplicações e limitações do mesmo.

Assim, baseando-se em resultados preliminares já realizados com ambos os métodos por Silva et al (2010 -a, 2010 -b; 2011 -a; 2011 -b), optou-se pela continuidade da modelagem do problema de transporte colaborativo deste artigo através do método SD. A opção por este método em detrimento ao método ABMS, foi conseqüência de dois fatores principais. O primeiro deles foi o fato do problema em estudo ser marcado pela complexidade dinâmica onde as ações de alguns agentes provocam reações em outros agentes. O segundo fator foi da necessidade de simular quantitativamente as políticas propostas para que pudesse ser avaliado o seu impacto nas metas dos agentes envolvidos.

O surgimento do SD deu-se em função de lidar com problemas caracterizados pela complexidade dinâmica, isto é, sistemas onde as ações de um determinado agente geram reações em outros agentes, também chamado de feedbacks (SANCHES, 2009). Conceitualmente, o conceito de feedback é o cerne da abordagem do System Dynamics. Intuitivamente, um loop de feedback existe quando uma informação resultante de alguma ação percorre o sistema e eventualmente retorna de alguma maneira a seu ponto de origem, potencialmente influenciando uma ação futura. Se a tendência do loop é reforçar a ação inicial, é chamado de positivo ou de reforço; se a tendência é opor-se à ação inicial, é chamado de negativo ou de balanço. O sinal do loop é chamado de polaridade (STERMAN, 2000).

Na continuidade deste trabalho, durante os anos de 2010 e de 2011 foram realizadas entrevistas empresários brasileiros, de diversos ramos (Weg, Votorantim, Stanley Black \& Decker, Tigre, Tupy, Brasmar, Log-In, dentre outras), cujo objetivo principal desse contato foi obter 
informações sobre o mecanismo de exportação praticado no mercado, verificar quais os principais agentes envolvidos neste processo e, especular se já há indícios de colaboração na realização do transporte marítimo e como os empresários brasileiros avaliam a possibilidade de adoção do CTM em suas operações. Além disso, buscou-se compreender melhor as práticas adotadas no mercado, para então modelar-se o problema de estudo, semelhante às práticas reais adotadas.

\subsection{Comportamento das indústrias}

O porto de partida da modelagem se deu utilizando-se do software Vensim ${ }^{\circledR}$ da empresa Ventana Systems, Inc. (versão DSS), ao se modelar através de um diagrama de estoque-fluxo, o comportamento das indústrias. O número acumulado de indústrias na colaboração ( Ind ) é expresso pelo número inicial de indústrias $\left(\operatorname{Ind}\left(\mathrm{t}_{0}\right)\right)$ somado à integração da taxa de variação de indústrias $(\dot{I})$ no tempo, conforme a equação (1):

$$
\operatorname{Ind}(t)=\operatorname{Ind}\left(t_{0}\right)+\int_{t_{0}}^{t} \dot{I} d t
$$

A variável $\dot{I}$ é expressa pelo número de ingresso de novas indústrias (ini) e o número de desistência de indústrias (di) no tempo $t$, conforme a equação (2):

$$
\dot{I}=i n i-d i \text {. }
$$

A variável ini varia de acordo com a razão de lucro ( $r a l$ ) obtida num determinado tempo $t$. Por ral entende-se a relação entre o preço de frete colaborativo ( Pref ) obtido e valor de frete mínimo praticado no mercado ao negociá-lo individualmente:

$$
\text { ral }=\text { pref } / 100 \text {. }
$$

Assim, tem-se que:

$$
\text { ini }=\left\{\begin{array}{ll}
0, & r a l>1 \\
8,1079 \cdot \exp (-2,179 \cdot r a l), & r a l \leq 1
\end{array},\right.
$$

ou seja, se a razão de lucro for superior a 1, o preço de frete da colaboração é superior ao preço de frete individual a ser obtido pela indústria e, portanto não é viável o ingresso da uma nova indústria na colaboração.

O número esperado de $d i$ varia também em função da $r a l$ : se $r a l \leq 0.58$ então $d i=0$ senão,

$$
d i=0,0801 \cdot \exp (2,1502 \cdot r a l),
$$

ou seja, à medida que ral aumenta torna-se inviável às indústrias manterem-se na colaboração. 
Seguindo o princípio de que os preços praticados não crescem infinitamente, para Ind >100 , Pref $=55$, e em caso contrário será alterado conforme a equação (6):

$$
\text { Pref }=100,38 \cdot \exp (-0,006 \cdot \text { Ind }) \text {. }
$$

\subsection{Comportamento dos navios}

O número de navios $(N a v)$ é expresso pelo número inicial de navios $\left(N a v\left(\mathrm{t}_{0}\right)\right)$ somado à integração da taxa de variação de navios $(\dot{J})$ no tempo, conforme a equação $(7)$ :

$$
\operatorname{Nav}(t)=\operatorname{Nav}\left(t_{0}\right)+\int_{t_{0}}^{t} \dot{J} d t .
$$

A variável $\dot{J}$ é expressa pelo número de ingresso de novos navios (inn) e o número de desistência de navios $(d n)$ no tempo $t$, conforme a equação (8):

$$
\dot{J}=i n n-d n
$$

A variável inn varia de acordo com Pref. Desse modo se Pref $<30$ (valor adotado aleatoriamente), então inn $=0$, ou seja, nenhum navio tem interesse em ingressar no sistema; porém quando Pref $\geq 30$, o número de ingresso de navios no sistema ocorrerá de acordo com a equação (9):

$$
\text { inn }=0,0669 \cdot \exp (0,0441 \cdot \text { Pref }) .
$$

O valor esperado de $d n$ será nulo quando Pref $>80$ e irá aumentar à medida que Pref é reduzido, pois ao armador (representando a oferta) torna-se cada vez menos atrativa a permanência no mercado à medida que o preço é reduzido, influenciando a desistência, ou retirada de navios disponíveis no mercado, no intuito de reduzir a oferta e aumentar o preço de frete novamente. Assim, o valor esperado de $d n$ é dado por:

$$
d n=8,6679 \cdot \exp (-0,043 \cdot \text { Pref }) \text {. }
$$

Por fim, a curva de oferta define-se a partir do preço de frete, que é resultante do número de navios ofertados no mercado. Sendo assim, para Nav $<1$, Pref $=50$ e em caso contrário:

$$
\text { Pref }=55,206 \cdot \exp (0,006 \cdot N a v)
$$




\subsection{Colaboração das indústrias e disponibilidade de navios em função do preço de frete marítimo}

Dando continuidade na proposta de Silva et al (2011-b), que considera isoladamente o comportamento das indústrias e o comportamento da disponibilidade de navios, foi modelado um digrama de estoque-fluxo que permite analisar simultaneamente o comportamento do sistema de oferta-demanda de navios afetando a colaboração entre as indústrias manufatureiras. Como uma variável do tipo estoque é alterada apenas pelos fluxos de entrada e de saída (STERMAN, 2000), consideraram-se como fluxos de entrada, a taxa de demanda (tdem) e a taxa de interesse de oferta (tof), representando a demanda e oferta, respectivamente. A tdem expressa o número de indústrias (Ind) existentes num dado período, porém este valor é convertido em número de navios (Nav), a fim de tratar de uma mesma unidade na análise. Dessa forma, tdem $=$ Ind $/ 3,33$ e tof $=$ Nav. Ver Figura 2.

Neste diagrama proposto a variável $d n$ assume os seguintes valores, conforme a equação (12):

$$
d n(\text { tof }, \text { tdem })=\left\{\begin{array}{l}
2, \text { tof } / \text { tdem } \geq 1, \\
1, \text { tof } / \text { tdem }<1
\end{array} .\right.
$$

Esta relação indica que se a taxa de oferta for maior ou igual à taxa de demanda num dado período de tempo $t, 2$ unidades de navios deixarão o sistema, no intuito de tentar reduzir o preço de frete. Em caso contrário, quando a taxa de demanda for superior à taxa de oferta, apenas 1 navio deixa o sistema (pois considerou-se que sempre pode haver algum desistente devido a outros interesses, mesmo até quando o negócio é viável).

Além destas considerações, Pref é expressa pelo valor inicial do preço de frete $\left(\operatorname{Pref}\left(\mathrm{t}_{0}\right)\right)$ somado à integração da taxa de variação do valor do frete $(\dot{M})$ no tempo, conforme a equação (13):

$$
\operatorname{Pref}(t)=\operatorname{Pref}\left(t_{0}\right)+\int_{t_{0}}^{t} \dot{M} d t
$$

onde, a variável $\dot{M}$ é regulada por tof e tdem, conforme a equação (14):

$$
\dot{M}=\left\{\begin{array}{l}
-((t o f-t d e m) \cdot 0,3), \text { tof }>\text { tdem } \\
((\text { tdem }- \text { tof }) \cdot 0,3), \text { tof } \leq \text { tdem }
\end{array} .\right.
$$

Deste modo, se a oferta for superior à demanda num determinado tempo $t$, o preço de frete sofrerá uma redução em seu valor, em caso contrário, o preço de frete sofrerá um aumento, no intuito de equilibrar o mercado, buscando-se a condição onde tof $=$ tdem . 
Figura 2 - Diagrama de estoque-fluxo das indústrias e navios

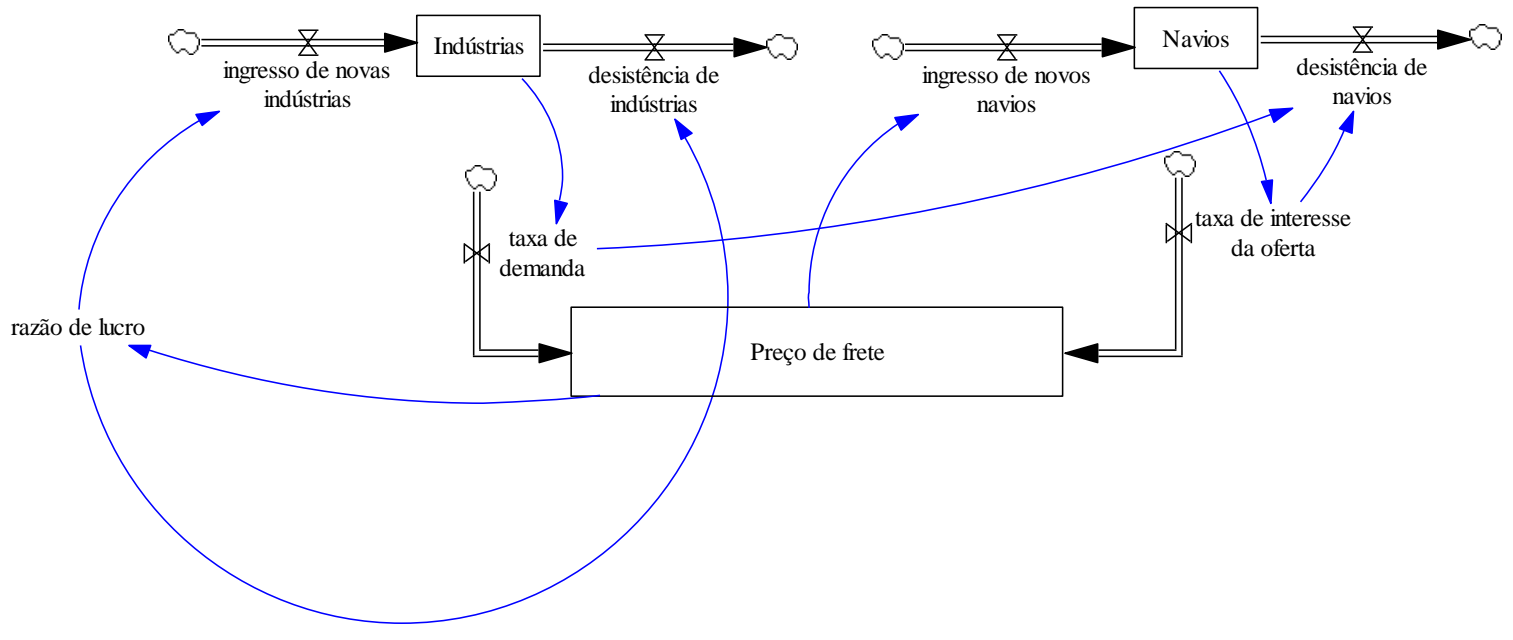

Fonte: Autoria própria

\subsection{Capacidade do hinterland}

De acordo com Novaes et al (2012) as áreas portuárias ocasionalmente sofrem com diversos problemas críticos que contribuem para os altos custos logísticos, sendo que um desses problemas é a falta de capacidade portuária. Assim, considerando que as operações de transporte marítimo envolvem também a necessidade de uma área (pátio) disponível no porto para recebimento e armazenamento de contêineres, conhecida como hinterland, buscou-se incluir tal variável no modelo. Sendo assim, Capacidade hinterland, foi modelada como sendo uma variável do tipo estoque, identificada por CapHin:

Assim, CapHin é expressa pelo valor inicial da capacidade do hinterland $\left(\operatorname{CapHin}\left(t_{0}\right)\right)$ somada à integração da taxa amp no tempo, conforme a equação (15):

$$
\operatorname{CapHin}(t)=\operatorname{CapHin}\left(t_{0}\right)+\int_{t_{0}}^{t} \operatorname{amp} d t,
$$

onde a variável amp (ampliação de área) é expressa, num primeiro momento como:

$$
a m p=\left\{\begin{array}{l}
300.000, \text { toc } \geq 0.75 \\
0, \text { toc }<0.75
\end{array},\right.
$$

Na concepção de Manne $(1961,1967)$ a expansão da capacidade consiste do processo de adição de instalações no decorrer do tempo de modo a satisfazer a demanda crescente, sendo que as decisões sobre expansão da capacidade geralmente resultam em um "forte" investimento de capital. Assim, calcula-se o VPL - Valor Presente Líquido dos investimentos, segundo equação dada por Casarotto e Kopittke (2010). De acordo com Novaes et al (2012), uma característica importante da 
maioria dos problemas de capacidade é o reconhecimento de economias de escala; ou seja, grandes instalações geralmente custam menos por unidade produzida do que as pequenas instalações. Neste sentido o termo "curva de aprendizagem" (COUTO e TEIXEIRA, 2005) é utilizada, conforme a expressão matemática:

$$
I^{(m)}=I^{(1)} \cdot m^{-\theta},
$$

onde $I^{(1)}$ é o valor do custo de construção da primeira unidade, $I^{(m)}$ é o valor do investimento na $m$ ésima unidade, e $\theta$ é a elasticidade do custo de construção em relação ao número de unidades construídas em sequência.

\subsection{Incentivo à exportação}

No Brasil o comércio exterior tem sido pouco utilizado como fator pró-ativo da estratégia do desenvolvimento e, sendo assim, decidiu-se incluir no modelo proposto, uma variável auxiliar que represente o incentivo ao aumento das exportações, denominada incentivo à exportação (incexp) a fim de avaliar o impacto gerado pelas indústrias ingressantes no sistema de transporte colaborativo. Desta maneira, a inserção da variável incexp se dá à variável ini, expressa pela equação (4), que por sua vez passa a ser definida como:

$$
\text { ini }=8,1079 \cdot \exp (-2,179 \cdot r a l)+\text { incexp } .
$$

\subsection{Impacto da indústria marítima}

Ao se modelar o comportamento colaborativo das indústrias em relação ao transporte marítimo, não se pode deixar de considerar o impacto que a indústria marítima pode gerar sobre tal sistema. O valor da discrepância existente na frota marítima, discrep, pode ser interpretado como sendo a diferença entre tdem e tof:

$$
\text { discrep }=\text { tdem }- \text { tof } \text {. }
$$

A variável discrep tem o papel de informar o valor do número de encomendas de navios (encnav) a serem realizadas por período de tempo. Portanto:

$$
\text { encnav }=\operatorname{Max}\{0, \text { discrep }\} \text {. }
$$

A variável encnavé definida como sendo um fluxo de entrada alimentador da variável Cartec. Esta, na condição de variável do tipo estoque, representa a carteira de encomendas e construção existente; ou seja, o número acumulado de navios que estão em construção a cada 
instante; que também é influenciada pelo fluxo de saída de entrega de navios (entnav). A equação (21) define Cartec:

$$
\operatorname{Cartec}(t)=\operatorname{Cartec}\left(t_{0}\right)+\int_{t_{0}}^{t} \dot{Z} d t,
$$

sendo $\operatorname{Cartec}\left(t_{0}\right)$, o valor inicial dessa carteira e $\dot{Z}$, a taxa de variação (no tempo) dos navios em construção. A variável $\dot{Z}$ é expressa pela diferença entre o número de encomendas de navios e o número de entrega de navios no tempo $t$, conforme a equação (22):

$$
\dot{Z}=\text { encnav-entnav. }
$$

Por se tratar a construção de navios, um trabalho moroso (tempo médio de construção, tmc), deve-se considerar a existência de um atraso entre o momento da encomenda de um navio e o momento de entrega do mesmo, e a variável entnav pode ser equacionada como:

$$
\text { entnav }=\frac{\text { encnav }}{\text { tmc }}
$$

sendo que além de ser um fluxo de saída de Cartec, também é reconhecida como sendo um fluxo de entrada da variável Nav, anteriormente definida no item 0. Esta, por sua vez, também é influenciada pelo fluxo de saída de demolição de navios (demnav), o qual representa a taxa com que são demolidos navios em cada período de tempo $t$. Para critério de simplificação do problema, são desconsiderados os motivos pelos quais ocorrem as demolições e adota-se demnav $=N a v * 0.05$ ; ou seja, a taxa de demolição adotada é de $5 \%$ do valor dos navios existentes.

Posto isso, o novo valor de Nav, anteriormente expresso pela equação (7), é dado por:

$$
N a v=\operatorname{Max}\{(\text { entnav }+i n n-d n-d e m n a v), 0\}
$$

ou seja, o maior valor entre 0 e a diferença entre a entrega, o ingresso, a desistência e a demolição de navios.

\section{Resultados}

Para avaliar o comportamento do modelo proposto, construiu-se um estudo com dados empíricos. A análise do problema em estudo prestou-se para demonstrar a funcionalidade do modelo e também avaliá-lo em relação à sensibilidade de suas variáveis. Quanto aos resultados obtidos com a simulação pode-se assumir que foram satisfatórios, pois permitiram gerar conclusões sobre o comportamento do sistema de transporte colaborativo marítimo. As conclusões obtidas são as seguintes: 
- À medida que o valor do preço de frete se eleva, eleva-se o número de ingresso de novas indústrias no mercado, uma vez que os armadores desejam maximizar suas receitas, oriundas do afretamento de navios e, portanto, poucos navios abandonam o sistema e quando a razão entre a taxa de interesse de oferta e a taxa de demanda for maior ou igual a 1, a desistência de navios passa a ser maior.

- O excesso de oferta de navios no mercado faz com que o preço de frete seja reduzido, uma vez que as indústrias em colaboração têm maior poder na negociação do valor do mesmo. O preço de frete, portanto reduz-se até o ponto em que começam a faltar navios no mercado, quando a razão entre a taxa de interesse de oferta e a taxa de demanda for menor que 1 , e então os armadores começam a aumentar novamente o valor do preço de frete, repetindo-se o ciclo ao longo do tempo, até que se alcance o ponto de equilíbrio.

- A capacidade do hinterland precisa ser adequada à demanda existente e sempre é preciso uma análise de viabilidade de investimento, no intuito de se determinar o melhor retorno financeiro (melhor VPL); levando-se em conta o instante $(t)$ a ser investido, bem como, as quantidades de ampliações a serem realizadas de modo a obter ganhos com economia de escala.

- No decorrer de um estudo sobre transporte é preciso atentar para a possibilidade de surgimento de outras variáveis exógenas, que na grande maioria das vezes desregulam o sistema. No caso analisado a variável de incentivo à exportação, contribui para o ingresso de novas indústrias, elevando bruscamente a taxa de demanda. Isso faz com que o gap entre a taxa de demanda e a taxa de interesse de oferta se eleve, acarretando acréscimos no preço de frete. Além disso, um incentivo à exportação ocasiona congestionamento na área do hinterland, necessitando de nova ampliação da capacidade, que não havia sido considerada no plano original.

- A indústria marítima busca reduzir a discrepância de frota ao suprir a demanda existente por navios. Realizam-se encomendas de construção de navios e à medida que o tempo avança, inicia-se a entrega dos mesmos. Como o tempo de construção de um navio adotado é de 16,7 meses, há um atraso entre o instante da encomenda e o instante da entrega. Os navios entregues fazem parte do estoque de navios disponíveis e interferem diretamente na taxa de interesse de oferta de navios. Quando a taxa de interesse de oferta é superior à taxa de demanda, o preço de frete reduz-se e praticamente cessa a taxa de ingresso de novos navios. A redução do preço de frete é condição ideal para que novas indústrias ingressem na colaboração, elevando a taxa de demanda acima da taxa de interesse de oferta. Tal condição atua na elevação do preço de frete e no ingresso de novos navios, além de acionar a indústria marítima novamente, repetindo-se o ciclo. 


\title{
6. Considerações sobre os resultados obtidos
}

Partindo da simplificação do problema em estudo, o modelo proposto (apesar de adotar relações empíricas), permitiu a realização de diversas simulações e a compreensão do funcionamento dinâmico do sistema de transporte colaborativo marítimo. Também foi possível compreender a importância isolada de cada variável além dos impactos gerados no sistema após a alteração dessas variáveis, o que se deu através de análises de sensibilidade. Após a realização do levantamento bibliográfico, pesquisa de campo junto a empresários, modelagem do problema e análise dos resultados numéricos do comportamento do sistema, identificou-se explicitamente e implicitamente benefícios oriundos do sistema de transporte colaborativo marítimo: custos administrativos rateados, fretes marítimos reduzidos, maior free-time de embarque, aumento no prazo para pagamento do frete, maior influência sobre os transportadores, fluxos constantes de importação e exportação, maior oferta de serviços por parte dos armadores e terminais, melhoria da área retroportuária, dentre outros.

Uma variedade de assuntos relacionados ao tema deste artigo não foi abordada no decorrer do trabalho existindo, portanto, diversas oportunidades para a continuidade do mesmo: expandir o modelo proposto ao incluir os demais agentes participantes na cadeia de exportação marítima, conforme apresentado na Figura 1, bem como aprimorar o detalhamento do comportamento dos mesmos, repetir o estudo após obter maior disponibilidade de dados quantitativos reais e calibração dos mesmos, efetuar o estudo em outros segmentos de indústrias no Brasil a fim de compará-los, propor um indicador de colaboração, aperfeiçoar o estudo sobre SD de modo a gerar melhores análises dos modelos de formação de colaboração. Com isso espera-se que seja indicada a formação de redes colaborativas no intuito de melhorar a eficiência da logística e o resultado econômico das empresas, como de fato propõe a abordagem de transporte colaborativo.

\begin{abstract}
This paper aims to present a model to analyze the systemic effects arising from the collaboration policies among the manufacturing industries, which use the maritime transportation to execute the exportation. It was researched about the Collaborative Logistics era and the Collaborative Transportation Management and, in order to validate the study proposal and to obtain data to insert in the model, some interviews were executed to entrepreneurs and specialists. Subsequently, it was performed a study about Agent Based Modeling and Simulation and System Dynamics. The proposed model using SD contributed to the analysis of the systemic effects arising from the collaboration policies among the manufacturing industries, which strengthen the bargain power if acting allied to each other, and has the power to influence the maritime freight rate reduction. This work contributed to the comprehension of the importance of adopting an interdisciplinary approach to deal with the maritime transportation problems.
\end{abstract}

Key-words: Logistics, Collaborative maritime transportation, System Dynamics, Simulation. 


\section{Agradecimentos}

Os autores agradecem ao CNPq e Capes, que financiaram este trabalho na forma de bolsa de estudo e em projetos.

\section{Referências}

BLOOS, MELANIE; KOPFER, HERBERT (2009) On the formation of operational transport collaboration systems. Annals of the II LogDynamics International Conference (LDIC): 329-338. Bremen, Alemanha, 17-21 de agosto.

CARNIERI, CELSO; SIMIEMA, HÉLIO HIPÓLITO; MAZZAROTTO, MARCO ANDRÉ (1983) Programa Integrado de Transporte de Soja. Anais do XVI Congresso da SBPO, Florianópolis.

CASAROTTO FILHO, NELSON C.; KOPITTKE, BRUNO H. (2010) Análise de investimentos. 11 ed. Atlas: São Paulo.

COUTO, J. P.; TEIXEIRA, J. C. (2005) Using linear model for learning curve effect on highrise floor construction. Construction Management and Economics, v. 23, p. 355-364.

\section{cross ref}

DRUCKER, PETER (1954) The Practice of management. Harper \& Row, Inc.: New York, NY, p.225.

GIESEN, RICARDO; MUÑOZ, JUAN CARLOS; SILVA, MARIELA; LEVA, MABEL (2007) Método de solución al problema de ruteo e inventarios de múltiples productos para uma flota heterogénea de naves. Actas del Congreso Chileno de Ingeniería de Transportes.

GOMBER, P; SCHMIDT, C; WINHARDT, C. (1997) Elektronische Märkte für die dezentrale Transportplanung. Wirstschaftsinformatik, 39 (2):137-145.

MANNE, A. S. (1961) Capacity expansion and probabilistic growth. Econometrica, v. 29, p. 632-649. cross ${ }^{\text {ref }}$

MANNE, A. S. (1967) Investment for capacity expansion: size, location and timephasing. Allen and Unwin: London.

NOVAES, ANTÔNIO GALVÃO N. (2007) Logística e gerenciamento da cadeia de distribuição. $3^{a}$ ed. Campus: Rio de Janeiro.

NOVAES, ANTONIO G. N; FRAZZON, ENZO M; BURIN, PAULO J. (2009) Dynamic vehicle routing in over congested urban areas. Annals of the II LogDynamics International Conference (LDIC): 103-112. Bremen, Alemanha, 17-21 de agosto.

NOVAES, ANTONIO G. N.; SCHOLZ-REITER, BERND; SILVA, VANINA MACOWSKI DURSKI; ROSA, HOBED (2012) Long-term planning of a container terminal under demand uncertainty and economies of scale. Pesquisa Operacional., 32, 1, 55-85. 
SANCHES, LARS MEYER (2009) Análise do acúmulo da demanda logística no final do período de comercialização: um modelo de Dinâmicas de Sistema para o setor de bens de consumo brasileiro. Tese de doutorado do Programa de Pós-Graduação da Faculdade de Engenharia Civil, Arquitetura e Urbanismo, da Universidade Estadual de Campinas.

SCHÖNBERGER, J. (2005) Operational freight carrier planning. Springer: Berlin, Heidelberg, New York.

SEIFERT, DIRK (2003) Collaborative Planning, Forecasting and Replenishment: how to create a supply chain advantage. Amacom: USA.

SILVA, VANINA MACOWSKI DURSKI; COELHO, ANTÔNIO SÉRGIO; ZAGO, CAMILA AVOZANI (2009) Collaborative Transportation Management (CTM): uma nova ferramenta de apoio à cadeia de suprimentos. Revista Tecnologia (UNIFOR), v. 30, p. 265-272.

SILVA, VANINA MACOWSKI DURSKI; NOVAES, ANTÔNIO GALVÃO; SCHOLZ-REITER, BERND; PIOTROWSKI, J. (2010 -a) Introducing the concept of Autonomous Agent as a tool to assist the analysis of the collaboration between manufactured exporters and maritime shippers. Proceedings of the 1st International Conference on Logistics and Maritime Systems (LOGMS). September, Pusan, South Korea.

SILVA, VANINA MACOWSKI DURSKI; LOUREIRO, SÉRGIO ADRIANO; NOVAES, ANTÔNIO G.; ORLANDO FONTES LIMA JR.; ANTÔNIO SÉRGIO COELHO (2010 -b) The Collaborative Maritime Transportation's problem under System Dynamics and Agent Based Modelling and Simulation approaches. Revista Tecnologia (UNIFOR), v. 31, n. 2 , in press.

SILVA, VANINA MACOWSKI DURSKI; LOUREIRO, SÉRGIO A.; NOVAES, ANTÔNIO. G. N.; SCHOLZREITER, BERND. (2011-a) Kollaborativer Seetransport: Beziehungspolitik der brasilianischen Exportkette für Fertigungsprodukte. Industrie Management 27:23-26.

SILVA, VANINA MACOWSKI DURSKI; COELHO, ANTÔNIO SÉRGIO; NOVAES, ANTÔNIO G. (2011-b) The role of collaboration through manufactured goods' exportation process under System Dynamics analysis. Proceedings of the 29th International Conference of The System Dynamics Society, July 24-28. Washington DC, USA.

SILVA, VANINA MACOWSKI DURSKI; COELHO, ANTÔNIO SÉRGIO; NOVAES, ANTÔNIO GALVÃO; LIMA JR, ORLANDO FONTES $(2011-\mathrm{c})$ Remarks on collaborative maritime transportation's problema using System Dynamics and Agent Based Modeling and Simulation approaches. In: Luis M. Camarinha-Matos; Alexandra PereiraKLEN; HAMIDEH AFSARMANESH (Eds.). Adaptation and Value Creating Collaborative Networks. 1 ed., p. 245-252, Springer .

STERMAN, JOHN (2000) Business dynamics: systems thinking and modeling for a complex world. McGraw-Hill Companies: USA.

STERMAN, JOHN (2002) All Models are wrong: reflections on becoming a system scientist. System Dynamics Review - vol. 18. 501-531. 
SUTHERLAND, JOEL (2003) Collaborative transportation management - creating value trough increased transportation efficiencies. Business Briefing - Pharmagenerics, September.

TACLA, DOUGLAS (2003) Estudo de transporte colaborativo de cargas de grande volume, com aplicação em caso de soja e fertilizantes. Tese de Doutorado. Programa de Engenharia Naval e Oceânica, área de concentração: Transportes e Sistemas Logísticos, Escola Politécnica da Universidade de São Paulo.

\section{Dados dos autores:}

Nome completo: Vanina Macowski Durski Silva

Filiação institucional: Universidade Tecnológica Federal do Paraná - UTFPR

Departamento: Engenharia de Produção

Função ou cargo ocupado: Professora

Endereço para correspondência: Avenida Monteiro Lobato, s/n, km 04 - Jardim Carvalho Ponta Grossa - PR 84016-210, Brasil

Telefones para contato: (42) 9926-4558

e-mail: vaninasilva@utfpr.edu.br

Nome completo: Antonio Sérgio Coelho

Filiação institucional: Universidade Federal de Santa Catarina - UFSC

Departamento: Engenharia de Produção

Função ou cargo ocupado: Professor

Endereço para correspondência: Campus Universitário Reitor João David Ferreira Lima, Trindade Florianópolis - Santa Catarina - Brasil, CEP: 88040-900

e-mail: coelho@deps.ufsc.br

\section{Nome completo: Antonio Galvão Novaes}

Filiação institucional: Universidade Federal de Santa Catarina - UFSC

Departamento: Engenharia de Produção

Função ou cargo ocupado: Professor

Endereço para correspondência: Campus Universitário Reitor João David Ferreira Lima, Trindade Florianópolis - Santa Catarina - Brasil, CEP: 88040-900

e-mail:novaes@deps.ufsc.br 